\title{
ANÁLISE MULTIVARIADA DE PARÂMETROS FÍSICO-QUÍMICOS EM AMOSTRAS DE VINHOS TINTOS COMERCIALIZADOS NA REGIÃO METROPOLITANA DO RECIFE
}

\author{
Marcelo Farias de Andrade, Dayvson José Palmeira de Souza, João Bosco Paraíso da Silva e Ana Paula Silveira Paim* \\ Departamento de Química Fundamental, Centro de Ciências Exatas e da Natureza, Universidade Federal de Pernambuco, \\ Av. Prof. Luiz Freire, s/n, 50740-550 Recife -PE, Brasil \\ Recebido em 14/2/07; aceito em 19/7/07; publicado na web em 19/12/07

\begin{abstract}
MULTIVARIATE ANALYSIS OF PHYSICO-CHEMICAL PARAMETERS IN SAMPLES OF RED WINES SOLD IN THE METROPOLITAN AREA OF RECIFE. The Brazilian legislation requires analysis of certain parameters to classify a wine and allow its commercialization. Some physico-chemical and some color parameters were determined in this work in samples of different red wines sold in the metropolitan area of Recife. Multivariate analysis comprising principal component analysis and hierarchical cluster analysis was employed to distinguish the analyzed wines. The results for $\mathrm{pH}$, chloride concentration, color parameters and ammonium content were the most important variables for sample classification. It was also possible to classify the wines as soft or dry wines and amongst the soft wines we could determine two out of four winegrowing producers.
\end{abstract}

Keywords: red wine; physico-chemical methods; chemometrics.

\section{INTRODUÇÃo}

O vinho é, por definição, a bebida obtida pela fermentação alcoólica do mosto simples de uva sã, fresca e madura. O mosto simples de uva é o produto obtido pelo esmagamento ou prensagem da uva, com a presença ou não de suas partes sólidas. Assim, é vedada a denominação de vinho para produtos obtidos de quaisquer outras matérias-primas ${ }^{1}$.

Sendo o vinho proveniente de um processo natural, que é a fermentação alcoólica, ele é elaborado ao invés de fabricado e possui composição química e sensorial distintas, de acordo com o solo, o clima e a água da região vinícola que origina as uvas.

Além dos fatores geográficos e tecnológicos, existem outros que também influenciam a qualidade de um bom vinho, tais como a garrafa na qual será armazenado (cor e forma), a rolha, a temperatura e a posição de estoque dos vinhos, as taças, a temperatura e a forma correta de serví-lo e, até mesmo, a combinação com as comidas. Tais fatores podem ser denominados como enológicos. Outros que merecem destaque especial são aqueles que os enólogos utilizam para definir o vinho como um alimento ao invés de uma simples bebida alcoólica: as vitaminas, os minerais, os aminoácidos, os carboidratos, ou seja, a sua composição química ${ }^{2,3}$.

No Brasil, o Ministério da Agricultura é o órgão responsável em criar e fiscalizar leis e normas que definem, regularizam e controlam a elaboração e circulação dos vinhos. A lei 10.970, de 12 de novembro de 2004, dispõe sobre produção, circulação e comercialização de vinhos $^{4}$ e a portaria 229 , de 25 de outubro de 1988 , decreta os padrões de identidade e qualidade do vinho ${ }^{5}$. Nesta portaria, são indicados, além das definições básicas dos diferentes tipos de vinho, os teores máximos e mínimos para alguns constituintes da composição do vinho.

Visando um controle adequado nas análises, a Organização Internacional da Uva e do Vinho - "Organisation Internationale de la Vigne et du Vin" - OIV e a Associação Oficial de Químicos Analíticos - "Association Official of Analytical Chemistry" - AOAC, estabeleceram procedimentos de análises específicos para a deter-

*e-mail: anaspaim@ufpe.br minação de alguns parâmetros em vinhos ${ }^{6}$.

Vários trabalhos buscam caracterizar os vinhos em função de sua constituição química. Muitas variáveis têm sido analisadas e estudos têm sido realizados para este fim, desde os que utilizam técnicas mais convencionais ${ }^{7-11}$, até aqueles que empregam espectroscopia de infravermelho ${ }^{12}$, Ressonância Magnética Nucle$\operatorname{ar}^{13}$, espectroscopia de massa com plasma indutivamente acoplado ${ }^{14}$, fluorescência de raios- $-\mathrm{X}^{15}$, entre outras.

Em função da grande quantidade de informações que a maioria destas técnicas costumam gerar, o emprego de técnicas quimiométricas multivariadas como Análise de Componentes Principais ("Principal Components Analysis - PCA") e Análise de Agrupamento Hierárquico ("Hierarchical Cluster Analysis - HCA") têm auxiliado na interpretação dos resultados obtidos ${ }^{16-19}$. Estas técnicas têm sido utilizadas com sucesso, por exemplo, na discriminação de vinhos brancos catalães ${ }^{20}$, bem como na classificação de outros produtos, como óleos de oliva sicilianos ${ }^{21}$. Em ambos os casos, um conjunto particular de parâmetros analíticos mostrou-se mais relevante em função das características locais.

Neste trabalho, foram determinados os parâmetros físico-químicos $(\mathrm{pH}$, acidez titulável total, densidade, teor alcoólico, cloretos, extrato seco total e cinzas), de coloração (densidade de cor, intensidade de cor, cor total dos pigmentos, cor dos pigmentos poliméricos, idade química e densidade ótica a $620 \mathrm{~nm}$ ) e a concentração de amônia em amostras de vinhos tintos comercializados na Região Metropolitana de Recife . Os parâmetros foram determinados com o objetivo de avaliar a possibilidade de classificação e diferenciação dos vinhos com auxílio das técnicas quimiométricas PCA e HCA.

\section{PARTE EXPERIMENTAL}

\section{Amostras}

Foram analisadas 5 diferentes marcas de vinhos tintos de mesa num total de 22 amostras de diferentes lotes, conforme mostra a Tabela 1. Os vinhos analisados foram: Botticelli (Cabernet Sauvignon - seco) produzido no Vale do São Francisco (Petrolina - 
PE); Carreteiro (suave) de Igaraçu (PE); Vinho do Frei (suave) de Vitória de Santo Antão (PE); Vinho Douro (suave) de Abreu e Lima (PE) e Vinho São Brás (suave) de São José de Mipibu (RN).

Tabela 1. Tipos, vinícolas e datas dos lotes das amostras de vinhos analisados

\begin{tabular}{lccc}
\hline Tipo & Vinícola & Amostra & Data Lote \\
\hline Seco & A & 1 & $10 / 2004$ \\
& & 2 & $01 / 2005$ \\
& & 3 & $03 / 2005$ \\
& B & 5 & $06 / 2005$ \\
& & NI \\
& & 7 & NI \\
& C & 9 & NI \\
& & 10 & NI \\
& & 11 & NI \\
& & 12 & $07 / 10 / 05$ \\
& & 13 & $07 / 10 / 05$ \\
& & 14 & $07 / 10 / 05$ \\
& D & 15 & $02 / 06 / 05$ \\
& & 16 & $14 / 01 / 05$ \\
& & 18 & $14 / 10 / 05$ \\
& & 19 & $21 / 09 / 05$ \\
& & 21 & $21 / 09 / 05$ \\
& & 21 & $30 / 11 / 05$ \\
& & 22 & $30 / 11 / 05$ \\
& & &
\end{tabular}

NI: Não informado na garrafa.

\section{Metodologia}

Os parâmetros físico-químicos: $\mathrm{pH}$, acidez titulável total (Att), densidade (DEN), teor alcoólico $(\mathrm{EtOH})$, cloretos $(\mathrm{NaCl})$, extrato seco total (Ext) e cinzas (Cnz) foram analisados de acordo com os métodos recomendados pela $\mathrm{AOAC}^{6}$. $\mathrm{O}$ teor de amônio $\left(\mathrm{N}_{-} \mathrm{NH}_{4}^{+}\right)$ foi determinado empregando análise por injeção em fluxo ${ }^{22}$. A determinação dos parâmetros referentes à coloração dos vinhos, densidade de cor (DCor), intensidade de cor (ICor), cor total dos pigmentos (CTP), cor dos pigmentos poliméricos (CPP), idade química (IQ) e densidade ótica a $620 \mathrm{~nm}$ (DO620), foi realizada ${ }^{23,24}$ usando um espectrofotômetro UV-Vis, Perkin-Elmer, modelo lambda 6 com cubeta de quartzo de caminho ótico de $10 \mathrm{~mm}$.

Os dados obtidos foram autoescalonados como forma de préprocessamento antes das análises por PCA e HCA. Na HCA utilizou-se o método de ligação completa entre os agrupamentos e como métrica a distância euclidiana. Para ambas as análises utilizou-se o software STATISTICA ${ }^{\circledR}$.

\section{RESULTADOS E DISCUSSÃO}

Os valores obtidos para $\mathrm{pH}$, acidez, teor alcoólico e amônio estão expressos na Tabela 2.

$\mathrm{O}$ valor médio de $\mathrm{pH}$ obtido foi de 3,98 para os vinhos secos e 3,30 para os vinhos suaves. Tais dados são condizentes com os encontrados na literatura para os vinhos secos do Rio Grande do Sul ${ }^{25,26}$ (3,8 a 4,0). Porém, acima dos encontrados em vinhos secos e suaves produzidos em Minas Gerais ${ }^{10}(3,44)$ e nos vinhos tintos espanhóis ${ }^{27}(3,60)$.

Dentre os principais ácidos existentes nos vinhos, os mais fortes são o tartárico, pirúvico e oxálico. Entretanto, na uva (e, portanto, no
Tabela 2. Valores de $\mathrm{pH}$, acidez titulável total (Att, como ácido tartárico), etanol (EtOH) e amônio (N-NH4+) obtidos para as amostras de vinhos tintos analisadas e seus respectivos desvios-padrão

\begin{tabular}{lcccc}
\hline Amostra & $\mathrm{pH}$ & $\begin{array}{c}\text { Att } \\
\left(\mathrm{g} \mathrm{L}^{-1}\right)\end{array}$ & $\begin{array}{c}\mathrm{EtOH} \\
(\% \mathrm{v} / \mathrm{v})\end{array}$ & $\begin{array}{c}\mathrm{N}^{-\mathrm{NH}_{4}}+ \\
\left(\mathrm{mg} \mathrm{L}^{-1}\right)\end{array}$ \\
\hline 1 & $3,96 \pm 0,02$ & $6,3 \pm 0,2$ & $10,1 \pm 0,1$ & $25,66 \pm 0,01$ \\
2 & $4,03 \pm 0,02$ & $5,3 \pm 0,2$ & $9,6 \pm 0,1$ & $29,69 \pm 0,01$ \\
3 & $3,87 \pm 0,02$ & $5,7 \pm 0,3$ & $9,2 \pm 0,1$ & $32,92 \pm 0,01$ \\
4 & $4,06 \pm 0,02$ & $5,7 \pm 0,2$ & $14,1 \pm 0,1$ & $29,42 \pm 0,01$ \\
5 & $3,29 \pm 0,02$ & $5,7 \pm 0,5$ & $9,5 \pm 0,1$ & $8,67 \pm 0,01$ \\
6 & $3,17 \pm 0,02$ & $3,8 \pm 0,3$ & $10,7 \pm 0,1$ & $10,75 \pm 0,01$ \\
7 & $3,16 \pm 0,02$ & $3,6 \pm 0,3$ & $11,0 \pm 0,1$ & $10,26 \pm 0,01$ \\
8 & $3,18 \pm 0,02$ & $3,7 \pm 0,3$ & $6,9 \pm 0,1$ & $9,89 \pm 0,01$ \\
9 & $3,56 \pm 0,02$ & $7,0 \pm 0,4$ & $11,4 \pm 0,1$ & $19,97 \pm 0,02$ \\
10 & $3,36 \pm 0,02$ & $4,4 \pm 0,3$ & $11,8 \pm 0,1$ & $20,03 \pm 0,02$ \\
11 & $3,28 \pm 0,02$ & $5,1 \pm 0,2$ & $13,6 \pm 0,1$ & $19,39 \pm 0,02$ \\
12 & $3,29 \pm 0,02$ & $5,0 \pm 0,2$ & $10,6 \pm 0,1$ & $20,91 \pm 0,02$ \\
13 & $3,28 \pm 0,02$ & $4,8 \pm 0,2$ & $9,7 \pm 0,1$ & $19,56 \pm 0,02$ \\
14 & $3,55 \pm 0,02$ & $4,0 \pm 0,2$ & $9,3 \pm 0,1$ & $34,12 \pm 0,03$ \\
15 & $3,70 \pm 0,02$ & $4,2 \pm 0,2$ & $7,2 \pm 0,1$ & $23,06 \pm 0,02$ \\
16 & $3,15 \pm 0,02$ & $5,0 \pm 0,3$ & $10,2 \pm 0,1$ & $26,43 \pm 0,02$ \\
17 & $2,98 \pm 0,02$ & $4,6 \pm 0,2$ & $10,1 \pm 0,1$ & $18,92 \pm 0,01$ \\
18 & $3,12 \pm 0,02$ & $4,9 \pm 0,2$ & $8,0 \pm 0,1$ & $18,82 \pm 0,01$ \\
19 & $3,13 \pm 0,02$ & $5,0 \pm 0,2$ & $6,9 \pm 0,1$ & $37,21 \pm 0,03$ \\
20 & $3,37 \pm 0,02$ & $7,0 \pm 0,2$ & $11,0 \pm 0,1$ & $16,23 \pm 0,01$ \\
21 & $3,38 \pm 0,02$ & $8,2 \pm 0,2$ & $11,2 \pm 0,1$ & $14,14 \pm 0,01$ \\
22 & $3,37 \pm 0,02$ & $7,9 \pm 0,2$ & $12,5 \pm 0,1$ & $16,68 \pm 0,01$ \\
\hline & & & &
\end{tabular}

vinho) existem em maior quantidade o tartárico, málico e cítrico. Portanto, como o ácido tartárico é o ácido mais forte e que se encontra em maior concentração, a pequena variação do $\mathrm{pH}$ dos vinhos indica que a quantidade deste ácido é praticamente a mesma em todas as amostras analisadas. Por outro lado, os valores elevados da acidez nas amostras 9 e da 20 a 22 sugerem que possuem maior quantidade de ácidos fracos em relação às demais amostras de vinhos analisadas. Com exceção da amostra 5, os resultados indicam que os vinhos da vinícola $B$ encontram-se fora das especificações legais ${ }^{5}\left(55,0-130,0 \mathrm{mmol} \mathrm{L}^{-1}\right.$ em $\mathrm{H}^{+}$ou 4,2 - 9,8 g L $\mathrm{g}^{-1}$ como ácido tartárico).

Em relação ao teor alcoólico (EtOH), excetuando-se as amostras $8,15,18$ e 19, as demais enquadram-se nos limites indicados pela legislação vigente para vinhos tintos de mesa, que é de 8,6$14,0 \%(\mathrm{v} / \mathrm{v})$. Estes são valores próximos dos comumente encontrados em vinhos nacionais $\operatorname{secos}^{25,26}$ e suaves ${ }^{28}$, que variam em torno de $11 \%$, também próximos dos espanhóis ${ }^{29}$ com $12 \%$, no entanto, bem abaixo dos portugueses ${ }^{30}$ (18\% de teor alcoólico).

Os elevados teores de amônio, freqüentemente superiores a 20 mg L ${ }^{-1}$, para as amostras de vinhos da vinícola A (vinho seco) e D (vinho suave) provavelmente são resultado da fermentação maloláctica dos vinhos e do tipo de solo em que as uvas foram plantadas $^{31}$. Observa-se entre as amostras de vinhos suaves (vinícolas A e D), que houve grande variação na concentração de amônio, sugerindo que foram usados diferentes tipos de uvas ${ }^{31}$.

A Tabela 3 apresenta os valores obtidos e respectivos desviospadrão para cloretos, cinzas, extrato seco e densidade.

Quanto ao teor de cloretos, os maiores valores são para os vinhos da vinícola $\mathrm{A}$, sugerindo-se que estes permaneceram maior tempo na fermentação, ocasionando maior extração dos constituintes das uvas. Tal fato é corroborado observando-se a coluna que expressa o teor de cinzas, também em quantidades altas. Em ambas colunas, pode-se perceber grande dispersão dos dados para todos os vinhos, indicando possível falha no processo enológico ou gran- 
Tabela 3. Teores de cloretos $(\mathrm{NaCl})$, cinzas $(\mathrm{Cnz})$, extrato seco total (Ext) e densidade (DEN) e seus respectivos desvios-padrão para as amostras de vinhos analisadas

\begin{tabular}{|c|c|c|c|c|}
\hline Amostra & $\mathrm{NaCl}\left(\mathrm{g} \mathrm{L}^{-1}\right)$ & $\mathrm{Cnz}\left(\mathrm{g} \mathrm{L}^{-1}\right)$ & Ext $\left(\mathrm{g} \mathrm{L}^{-1}\right)$ & $\operatorname{DEN}\left(\mathrm{g} \mathrm{cm}^{-3}\right)$ \\
\hline 1 & $0,15 \pm 0,01$ & $4,12 \pm 0,06$ & $15,3 \pm 0,3$ & $0,99464 \pm 0,00005$ \\
\hline 2 & $0,12 \pm 0,01$ & $3,43 \pm 0,06$ & $14,7 \pm 0,2$ & $0,99255 \pm 0,00005$ \\
\hline 3 & $0,26 \pm 0,03$ & $3,30 \pm 0,03$ & $14,7 \pm 0,3$ & $0,99317 \pm 0,00007$ \\
\hline 4 & $0,21 \pm 0,01$ & $3,78 \pm 0,02$ & $15,6 \pm 0,2$ & $0,99565 \pm 0,00004$ \\
\hline 5 & $0,036 \pm 0,003$ & $1,80 \pm 0,02$ & $55,0 \pm 0,8$ & $1,01179 \pm 0,00005$ \\
\hline 6 & $0,062 \pm 0,003$ & $2,81 \pm 0,02$ & $56,4 \pm 0,2$ & $1,01704 \pm 0,00005$ \\
\hline 7 & $0,094 \pm 0,006$ & $2,17 \pm 0,02$ & $33,4 \pm 0,2$ & $1,01588 \pm 0,00005$ \\
\hline 8 & $0,090 \pm 0,006$ & $1,92 \pm 0,02$ & $49,7 \pm 0,2$ & $1,01797 \pm 0,00005$ \\
\hline 9 & $0,05 \pm 0,01$ & $1,38 \pm 0,02$ & $41,3 \pm 0,5$ & $1,02292 \pm 0,00005$ \\
\hline 10 & $0,05 \pm 0,01$ & $1,73 \pm 0,02$ & $56,3 \pm 0,2$ & $1,02337 \pm 0,00009$ \\
\hline 11 & $0,033 \pm 0,003$ & $4,54 \pm 0,02$ & $46,0 \pm 0,2$ & $1,02728 \pm 0,00008$ \\
\hline 12 & $0,026 \pm 0,003$ & $3,30 \pm 0,02$ & $68,2 \pm 0,2$ & $1,02766 \pm 0,00008$ \\
\hline 13 & $0,032 \pm 0,003$ & $4,40 \pm 0,02$ & $69,8 \pm 0,2$ & $1,02871 \pm 0,00008$ \\
\hline 14 & $0,047 \pm 0,003$ & $1,68 \pm 0,02$ & $28,0 \pm 0,3$ & $0,99963 \pm 0,00007$ \\
\hline 15 & $0,05 \pm 0,01$ & $3,14 \pm 0,04$ & $27,0 \pm 0,4$ & $1,00553 \pm 0,00007$ \\
\hline 16 & $0,121 \pm 0,006$ & $1,61 \pm 0,02$ & $27,6 \pm 0,2$ & $1,00617 \pm 0,00007$ \\
\hline 17 & $0,047 \pm 0,005$ & $2,74 \pm 0,02$ & $23,0 \pm 0,2$ & $1,01246 \pm 0,00008$ \\
\hline 18 & $0,041 \pm 0,005$ & $3,49 \pm 0,02$ & $23,5 \pm 0,2$ & $1,00926 \pm 0,00008$ \\
\hline 19 & $0,017 \pm 0,005$ & $3,07 \pm 0,02$ & $25,0 \pm 0,2$ & $1,00988 \pm 0,00008$ \\
\hline 20 & $0,023 \pm 0,003$ & $5,06 \pm 0,02$ & $31,8 \pm 0,2$ & $1,00303 \pm 0,00005$ \\
\hline 21 & $0,028 \pm 0,005$ & $2,24 \pm 0,02$ & $17,2 \pm 0,2$ & $1,00244 \pm 0,00005$ \\
\hline 22 & $0,033 \pm 0,006$ & $2,23 \pm 0,02$ & $20,9 \pm 0,2$ & $1,00287 \pm 0,00005$ \\
\hline
\end{tabular}

de diferença entre as uvas utilizadas nesta elaboração, inclusive para um mesmo fabricante.

No que se refere às especificações legais (máximo de $0,20 \mathrm{~g} \mathrm{~L}^{-1}$ como $\mathrm{NaCl}$ e 1,5 $\mathrm{g} \mathrm{L}^{-1}$ para cinzas ${ }^{5}$ ), apenas as amostras 3 e 4 não se enquadram frente ao teor de cloretos e apenas a amostra 9 está de acordo no que se refere ao teor de cinzas. Visto que mais de $95 \%$ das amostras se encontraram, acima do valor máximo estabelecido pela legislação para a quantidade de cinzas e que também há altos valores encontrados na literatura ${ }^{10,25,26,28}$, pode-se sugerir que tais limites não são mais adequados aos vinhos nacionais. Tal inferência também foi sugerida por Rizzon e Miele ${ }^{32}$ em seus estudos sobre extrato seco total dos vinhos nacionais.

Estudos realizados na Espanha ${ }^{29}$, em Portugal ${ }^{30}$ e na Itália ${ }^{33}$ também encontraram teores de cinzas maiores que $1,5 \mathrm{~g} \mathrm{~L}^{-1} \mathrm{em}$ seus vinhos.

Em relação ao extrato seco e densidade, observa-se clara distinção entre vinhos secos e suaves, tendo sido encontrados valores de densidade abaixo de $1 \mathrm{~g} \mathrm{~cm}^{-3}$ para amostras com menor teor de extrato seco. No que se refere aos vinhos suaves, as amostras de maior densidade apresentam também os maiores teores de extrato seco. Os valores elevados de densidade (maiores que $1 \mathrm{~g}$ $\mathrm{cm}^{-3}$ ) e de teor de extrato seco podem ser atribuídos à adição (indicada em seus rótulos) de açúcar em maior quantidade na composição desses vinhos.

A Tabela 4 expressa os valores obtidos nas análises dos parâmetros referentes à coloração dos vinhos.

A diferença observada para ICor indica maior quantidade de pigmentação natural nos vinhos secos em relação aos suaves. Para DCor, também chamada tonalidade, as amostras das vinícolas A e $\mathrm{D}$ apresentaram a razão flavonóides/antocianinas $\left(\mathrm{DO}_{420 \mathrm{~mm}} / \mathrm{DO}_{520 \mathrm{~mm}}\right)$ com maior variância que aquelas das vinícolas $\mathrm{B}, \mathrm{C}$ e E.

Observa-se para DO620 a mesma diferença entre vinhos secos e suaves, tal qual para ICor. Estes dois fatores apenas informam o que é claramente observado na análise visual dos vinhos, ou seja, a coloração mais intensa para o vinho seco e menos intensa para os suaves. Isto, provavelmente, é devido à maior quantidade de pigmentação natural existente nos vinhos secos.
Na literatura, encontrou-se apenas dados destas variáveis para vinhos secos, estando os resultados encontrados aqui próximos aos valores de outros vinhos nacionais ${ }^{28}$ para ICor $(5,20)$ e menores para DCor $(0,79)$.

A diferença observada nos parâmetros anteriores referentes à quantidade de pigmentos nos vinhos é mais pronunciada quando se analisa a coluna 5 da Tabela 4 , referente à cor total dos pigmentos, CTP. Observam-se valores cuja razão é até sete vezes, ou seja, existem cerca de sete vezes mais corantes naturais nos vinhos se$\cos$ que nos suaves.

Quanto à cor dos pigmentos na forma polimerizada, CPP, observa-se que os valores não variaram muito para as diferentes amostras. Merece destaque a grande concordância de valores (observada também em todos os outros parâmetros de coloração) para as amostras 20-22, pertencentes à vinícola E, sugerindo existir controle, mas não necessariamente qualidade, na elaboração do vinho por parte da vinícola.

Com relação aos resultados obtidos para a idade química (IQ) dos vinhos, vale inicialmente destacar que os valores de IQ para os vinhos secos são menores que aqueles dos vinhos suaves. Os nossos resultados encontram-se, portanto, condizentes com as datas dos lotes (Tabela 1) de algumas amostras, ou seja, vinhos mais "antigos" possuem um valor de IQ maior em relação aos mais "novos". Exceção observada para os vinhos da vinícola D, onde lotes de datas próximas $(15,18$ e 19$)$ geraram grande diferença em IQ. Por outro lado, para algumas amostras, não foi possível avaliar tal fato, pois não havia dados referentes ao lote nos rótulos das garrafas de vinhos que foram analisados.

\section{Análise quimiométrica}

A PCA mostra que $74,4 \%$ da informação contida nas Tabelas 2-4 pode ser representada em três componentes principais. $\mathrm{Na} \mathrm{Fi-}$ gura 1a é apresentado o gráfico dos escores de PC1 (com 41,7\% da variância) versus PC2 (com 20,6\% da variância). Nesta figura é possível notar que PC1 separa os vinhos secos, em valores de escores negativos, dos vinhos suaves em valores de escores positivos. 
Tabela 4. Valores obtidos nas determinações de intensidade de cor (Icor), densidade de cor (Dcor), cor total dos pigmentos (CTP), cor dos pigmentos poliméricos (CPP), idade química (IQ) e densidade ótica a $620 \mathrm{~nm}$ com seus respectivos desvios-padrão

\begin{tabular}{|c|c|c|c|c|c|c|}
\hline Amostras & Icor & Dcor & DO620 & CTP & $\mathrm{CPP}$ & IQ (\%) \\
\hline 1 & $3,74 \pm 0,02$ & $0,99 \pm 0,02$ & $1,342 \pm 0,002$ & $7,88 \pm 0,05$ & $1,37 \pm 0,02$ & $17,35 \pm 0,04$ \\
\hline 2 & $3,97 \pm 0,04$ & $0,89 \pm 0,03$ & $1,363 \pm 0,002$ & $8,11 \pm 0,04$ & $1,37 \pm 0,02$ & $16,92 \pm 0,03$ \\
\hline 3 & $3,60 \pm 0,04$ & $0,92 \pm 0,03$ & $0,977 \pm 0,002$ & $10,75 \pm 0,02$ & $1,37 \pm 0,02$ & $12,70 \pm 0,05$ \\
\hline 4 & $7,65 \pm 0,05$ & $0,87 \pm 0,03$ & $1,469 \pm 0,002$ & $12,03 \pm 0,02$ & $1,66 \pm 0,02$ & $13,81 \pm 0,05$ \\
\hline 5 & $2,64 \pm 0,02$ & $0,94 \pm 0,02$ & $0,452 \pm 0,002$ & $3,78 \pm 0,04$ & $1,35 \pm 0,02$ & $35,58 \pm 0,05$ \\
\hline 6 & $2,84 \pm 0,02$ & $0,92 \pm 0,02$ & $0,608 \pm 0,002$ & $5,74 \pm 0,02$ & $1,42 \pm 0,02$ & $24,65 \pm 0,04$ \\
\hline 7 & $2,75 \pm 0,02$ & $0,92 \pm 0,02$ & $0,603 \pm 0,002$ & $5,55 \pm 0,02$ & $1,42 \pm 0,02$ & $25,58 \pm 0,05$ \\
\hline 8 & $2,75 \pm 0,02$ & $0,92 \pm 0,02$ & $0,618 \pm 0,002$ & $5,61 \pm 0,02$ & $1,42 \pm 0,02$ & $25,33 \pm 0,05$ \\
\hline 9 & $2,62 \pm 0,02$ & $0,92 \pm 0,02$ & $0,627 \pm 0,002$ & $4,67 \pm 0,02$ & $1,42 \pm 0,02$ & $30,36 \pm 0,05$ \\
\hline 10 & $2,65 \pm 0,04$ & $0,93 \pm 0,03$ & $0,635 \pm 0,002$ & $4,94 \pm 0,02$ & $1,37 \pm 0,02$ & $27,63 \pm 0,04$ \\
\hline 11 & $2,75 \pm 0,02$ & $0,92 \pm 0,02$ & $0,735 \pm 0,002$ & $5,56 \pm 0,02$ & $1,44 \pm 0,02$ & $25,82 \pm 0,04$ \\
\hline 12 & $2,76 \pm 0,02$ & $0,92 \pm 0,02$ & $0,761 \pm 0,002$ & $5,87 \pm 0,02$ & $1,44 \pm 0,02$ & $24,49 \pm 0,04$ \\
\hline 13 & $2,76 \pm 0,02$ & $0,92 \pm 0,02$ & $0,601 \pm 0,002$ & $6,47 \pm 0,02$ & $1,44 \pm 0,02$ & $22,19 \pm 0,07$ \\
\hline 14 & $2,38 \pm 0,05$ & $1,04 \pm 0,04$ & $0,340 \pm 0,002$ & $1,71 \pm 0,02$ & $1,10 \pm 0,02$ & $64,04 \pm 0,05$ \\
\hline 15 & $2,56 \pm 0,02$ & $0,97 \pm 0,02$ & $0,427 \pm 0,002$ & $2,89 \pm 0,02$ & $1,23 \pm 0,03$ & $42,70 \pm 0,05$ \\
\hline 16 & $2,56 \pm 0,04$ & $0,93 \pm 0,03$ & $0,470 \pm 0,002$ & $3,89 \pm 0,02$ & $1,31 \pm 0,02$ & $33,60 \pm 0,05$ \\
\hline 17 & $2,77 \pm 0,02$ & $1,07 \pm 0,02$ & $0,458 \pm 0,002$ & $3,67 \pm 0,02$ & $1,33 \pm 0,03$ & $36,16 \pm 0,07$ \\
\hline 18 & $2,81 \pm 0,02$ & $1,05 \pm 0,02$ & $0,471 \pm 0,002$ & $4,86 \pm 0,02$ & $1,32 \pm 0,02$ & $27,21 \pm 0,07$ \\
\hline 19 & $2,61 \pm 0,02$ & $0,93 \pm 0,02$ & $0,479 \pm 0,002$ & $5,27 \pm 0,02$ & $1,33 \pm 0,02$ & $25,28 \pm 0,07$ \\
\hline 20 & $2,55 \pm 0,03$ & $0,80 \pm 0,02$ & $0,526 \pm 0,002$ & $5,35 \pm 0,02$ & $1,42 \pm 0,02$ & $26,57 \pm 0,08$ \\
\hline 21 & $2,54 \pm 0,02$ & $0,79 \pm 0,02$ & $0,512 \pm 0,002$ & $5,61 \pm 0,02$ & $1,42 \pm 0,02$ & $25,34 \pm 0,05$ \\
\hline 22 & $2,54 \pm 0,02$ & $0,79 \pm 0,02$ & $0,515 \pm 0,002$ & $5,31 \pm 0,02$ & $1,42 \pm 0,02$ & $26,80 \pm 0,05$ \\
\hline
\end{tabular}

PC2 por sua vez consegue separar, dentre os vinhos suaves, os da vinícola $\mathrm{D}$ (14-19) em valores de escores negativos dos demais das vinícolas $\mathrm{B}, \mathrm{C}$ e E, em valores de escores positivos.

Uma inspeção no gráfico dos "loadings", Figura 1b, mostra que a separação entre vinhos secos e suaves em PC1 é determinada, essencialmente, pelos conjuntos de parâmetros Ext, DEN e IQ (com "loadings" positivos) e CTP, NaCl, DO620, ICOR e pH (com
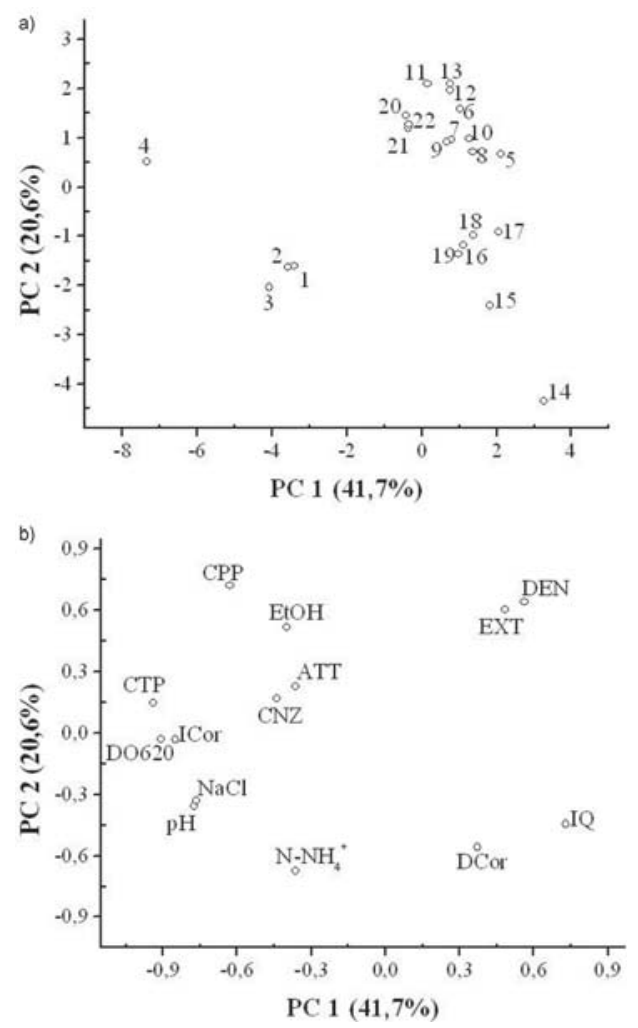

Figura 1. Gráfico dos: a) escores e b) loadings para PC1 x PC2 "loadings" negativos). Assim, por exemplo, os vinhos secos encontram-se mais à esquerda no gráfico dos escores (Figura 1a) por exibirem maior conteúdo de pigmentação natural e permanecerem com maior tempo de fermentação (maior extração de pigmentação e de cloretos e maior pH devido, possivelmente, à fermentação malolática). Por outro lado, a adição de açúcar, que implicará diretamente nos valores da densidade e do conteúdo de extrato seco total, justifica a disposição dos vinhos suaves à direita na Figura 1a. Analisando a Figura 1b, percebemos que PC2 é determinado, principalmente, pe-

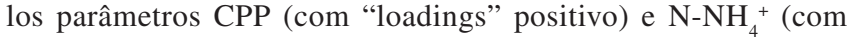
"loadings" negativo). Portanto, $\mathrm{PC} 2$ diferencia os vinhos suaves D (14-19) em função do seu maior teor de amônio e menor valor de CPP quando comparados aos demais vinhos suaves.

Na Figura 2a é apresentado o gráfico dos escores de $\mathrm{PC} 1$ versus PC3 (com 12,1\% da variância). Observa-se que PC3 consegue separar os vinhos suaves da vinícola E (20-22) dos demais vinhos. Uma análise do gráfico dos "loadings", Figura $2 b$, mostra que esta separação ocorre, principalmente, devido à maior acidez total dos vinhos desta vinícola.

A fim de avaliar os resultados obtidos pela PCA, realizamos uma HCA. O dendograma obtido é apresentado na Figura 3.

Nesta figura observa-se a presença de dois grandes agrupamentos, um menor associado às amostras de vinhos secos (1-4) e um outro maior associado aos vinhos suaves (5-22). Em ambos agrupamentos nota-se ainda que as amostras 4 e 14 são as últimas a se ligarem aos seus respectivos agrupamentos. Este resultado está em completa concordância com a disposição encontrada no gráfico dos escores da Figura 1a. Em particular, para o agrupamento relacionado aos vinhos suaves observa-se a presença de sub-agrupamentos relacionados às vinícolas $\mathrm{E}$, parte das amostras da $\mathrm{C}$, da $\mathrm{D}$ e uma mistura das amostras das vinícolas $\mathrm{C}$ e $\mathrm{B}$.

\section{CONCLUSÃO}

Nas amostras de vinhos tintos comercializados na Região Metropolitana do Recife, observou-se que o teor de cinzas de 95\% dos 

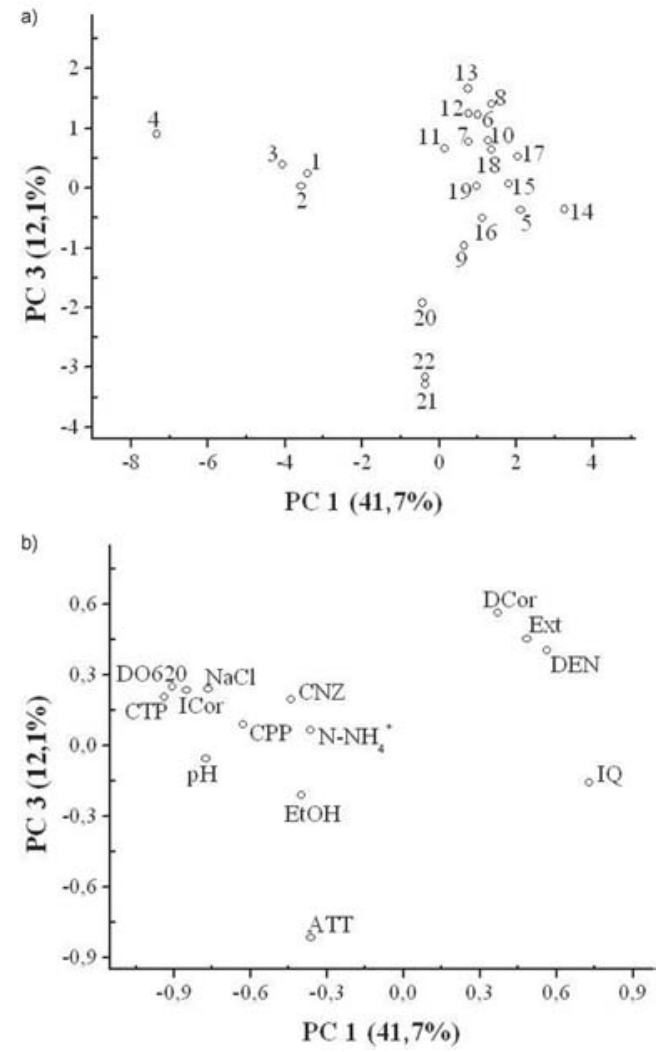

Figura 2. Gráfico dos: a) escores e b) loadings para PC1 x PC3

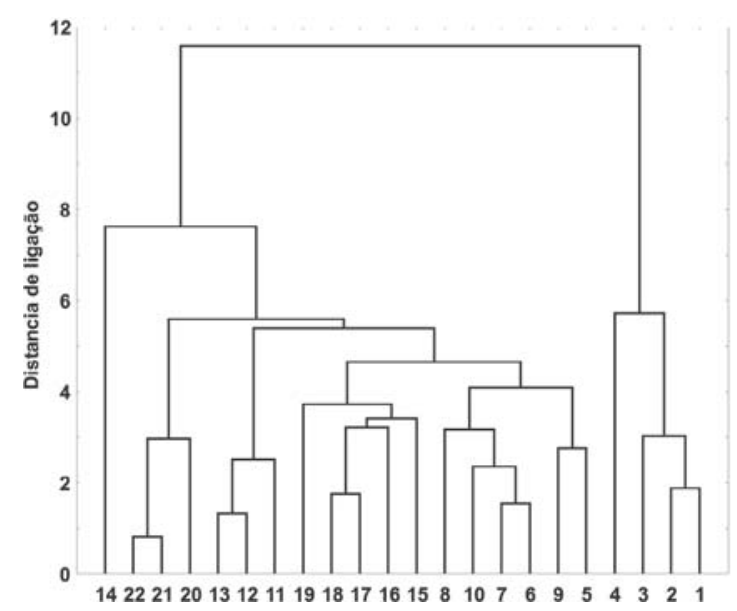

Figura 3. Dendograma para as 22 amostras de vinhos

vinhos analisados situou-se acima do limite permitido pela legislação nacional.

O teor de acidez encontrou-se fora dos padrões, indicando desequilíbrio na quantidade dos ácidos, possibilitando menor conservação do vinho.

A espectroscopia UV-Vis, em relação aos métodos clássicos de análises, apresentou-se mais rápida e eficaz (maior peso e quantidade de parâmetros) para a caracterização e distinção dos vinhos.

Análises quimiométricas de PCA e HCA foram capazes de distinguir os vinhos analisados quanto ao teor de açúcar em sua composição, se secos ou suaves, e a vinícola produtora. A técnica de PCA mostrou ainda que, dentre os parâmetros investigados, os de maior importância para distinção de vinhos secos e suaves, são as variáveis $\mathrm{pH}$, teor de cloretos, idade química, intensidade de cor, densidade ótica a $620 \mathrm{~nm}$, cor total dos pigmentos e cor dos pigmentos poliméricos. Já para distinção entre os vinhos suaves estão a cor dos pigmentos poliméricos, o teor de amônio e a acidez total.

Estudos estão em andamento no nosso laboratório para avaliar a importância de outros parâmetros (incluindo aqueles obtidos por outros métodos, como ${ }^{1} \mathrm{H}$ RMN e infravermelho próximo - NIR) para fins de discriminação, classificação e calibração dos parâmetros clássicos da AOAC.

\section{AGRADECIMENTOS}

Ao CNPq, FACEPE e CAPES, que apoiaram os trabalhos concedendo bolsas e auxílios à pesquisa. Ao Dr. G. Pereira (Embrapa Semi-árido), à Profa. Dra. M. F. Pimentel (DEQ-UFPE), à M. Sc. M. Lira (ITEP) e aos colegas da disciplina Trabalhos Dirigidos de Química liderados pelo Prof. Dr. A. M. Simas (DQF-UFPE) pelas valiosas sugestões.

\section{REFERÊNCIAS}

1. http://www.uvibra.com.br/legislacao_lei 7678.htm, acessada em Abril 2006.

2. Amarante, J. O. A.; Vinhos do Brasil e do Mundo, para conhecer e beber, $6^{\mathrm{a}}$ ed., Summus editorial Ltda.: São Paulo, 1983.

3. Pacheco, A. O.; Iniciação à Enologia, Ed. SENAC: São Paulo, 1995.

4. http://www.uvibra.com.br/legislacao_lei10970.htm, acessada em Abril 2006.

5. http://www.uvibra.com.br/legislacao_portaria229.htm, acessada em Abril 2006.

6. Association of Official Analytical Chemists; Official methods of analysis, $15^{\text {th }}$ ed., Washington, 1990.

7. Rizzon, L. A.; Miele, A.; Cienc. Rural 2006, 36, 959.

8. Rizzon, L. A.; Miele, A.; Cienc. Rural 2006, 36, 271.

9. Pilone, G. J.; Kunkee, R. E.; Am. J. Enol. Vitic. 1970, $21,128$.

10. Silva, T. G.; Regina, M. A.; Rosier, J. P.; Rizzon, L. A.; Chalfun, N. N. J.; Ciênc. Agrotec. 1999, 23, 632.

11. Rizzon, L. A.; Miele, A.; Ciênc. Tecnol. Aliment. 2004, 24, 223.

12. Palma, M.; Barroso, C. G.; Talanta 2002, 58, 265.

13. Brescia, M. A.; Caldarola, V.; De Glglio, A.; Benedetti, D.; Fanizzi, F. P.; Sacco, A.; Anal. Chim. Acta 2002, 458, 177.

14. Kment, P.; Mihaljevic, M.; Ettler, V.; Sebek, O.; Strnad, L.; Rohlová, L.; Food Chem. 2005, 91, 157.

15. Anjos, M. J.; Barroso, R. C.; Lopes, R. T.; de Jesus, E. F. O.; Simabuco, S. M.; Castro, C. R. F.; X-Ray Spectrom. 2004, 33, 407.

16. Kachigan, S. K.; Statistical Analysis. An Interdisciplinary Introduction to Univariate \& Multivariate Methods, Radius Press: New York, 1986.

17. Mardia, K. V.; Kent, J. T.; Bibly, J. M.; Multivariate Analysis, Academic Press: London, 1979.

18. Correia, P. R. M. ; Ferreira, M. M. C.; Quim. Nova 2007, 30, 481.

19. Bruns, R. E.; Faigle, J. F. G.; Quim. Nova 1985, 8, 84.

20. Larrechi, M. S.; Franques, M. R.; Ferre, M.; Rius, F. X.; J. Chemom. 1988, 3,261 .

21. Alberghina, G.; Caruso, L.; Fisichella, S.; Musumarra, G.; J. Sci. Food. Agric. 1991, 56, 445.

22. Nóbrega, J. A.; Mozeto, A. A.; Alberici, R. M.; Guimarães, J. L.; J. Braz. Chem. Soc. 1995, 6, 327.

23. Gómez-Plaza, E.; Gil-Muñoz, R.; López-Roca, J. M.; Martínez, A.; Food Res. Intern. 1999, 32, 503.

24. Bakker, J.; Preston, N. W.; Timberlake, C. F.; Am. J. Enol. Vitic. 1986, 37, 121.

25. Rizzon, L. A.; Miele, A.; Meneguzzo, J.; Zanuz, M. C.; Pesq. Agropec. Bras. 1999, 34, 1285.

26. Manfroi, V.; Miele, A.; Rizzon, L. A.; Barradas, C. I. N.; Manfrói, L.; Cienc. Rural 1997, 27, 139.

27. Darías-Martín, J.; Socas-Hernández, A.; Díaz-Romero, C.; Díaz-Díaz, E.; J. Food Comp. Anal. 2003, 16, 555.

28. Rizzon, L. A.; Miele, A.; Ciênc. Tecnol. Aliment. 2002, 22, 192.

29. Díaz, C.; Conde, J. E.; Claverie, C.; Díaz, E.; Trujillo, J. P. P.; J. Food Comp. Anal. 2003, 16, 49.

30. Nogueira, J. M. F.; Nascimento, A. M. D.; J. Agric. Food Chem. 1999, 47, 566.

31. Curvelo-Garcia, A. S.; Controlo de Qualidade dos Vinhos, Instituto da Vinha e do Vinho: Portugal, 1988.

32. Rizzon, L. A.; Miele, A.; Cienc. Rural 1996, 26, 297.

33. Moret, I. ; Scarponi, G.; Cescon, P.; J. Agric. Food Chem. 1994, 42, 1143. 